\title{
Spread of injectate in ultrasound-guided serratus plane block: a cadaveric study
}

Tatsuya Kunigo $^{1 *}$, Takeshi Murouchi ${ }^{2}$, Shuji Yamamoto ${ }^{3}$ and Michiaki Yamakage ${ }^{4}$

\begin{abstract}
Background: Serratus plane block is a thoracic truncal block that has been proposed as alternatives for analgesia such as epidural anesthesia and paravertebral block for the anterolateral chest wall. Previously, we performed the clinical study about optimal volume of the local anesthetic in serratus plane block. The primary aim of this study was to assess the pattern of distribution of dye into the serratus plane of cadavers after ultrasound-guided serratus plane injection.

Findings: Ultrasound-guided serratus plane injection was performed at the level of the fourth rib on the mid-axillary line in nine adult Thiel-embalmed cadavers. In each cadaver, one side was injected with $20 \mathrm{ml}$ of methylene blue dye and the contralateral side with $40 \mathrm{ml}$. Dissections of the thoracic walls were performed $20 \mathrm{~min}$ after the injection. The spread of the dye to intercostal nerves, lateral and medial pectoral nerves, long thoracic nerve, and thoracodorsal nerves was assessed. All T2-T5 intercostal nerves in the 40-ml group and all T3-T4 nerves in the 20-ml group were stained with the dye. A larger number of intercostal nerves was stained in the 40-ml group than that in the 20-ml group. Medial and lateral pectoral nerves were not frequently stained in either group.

Conclusions: The range of craniocaudal spread of the injectate was wider in the 40-ml group than that in the 20-ml group after ultrasound-guided serratus plane injection in Thiel-embalmed cadavers.
\end{abstract}

Keywords: Breast surgery, Cadaveric study, PECS block, Serratus plane block

\section{Introduction}

Pectoral nerve (PECS) block and serratus plane block are performed to suppress the pain in the anterior thoracic wall. Blanco et al. suggested that PECS I and II blocks and serratus plane block can be performed with ultrasound guidance [1-3]. The local anesthetic is injected between the pectoralis major and minor muscles for PECS I block, between the pectoralis minor and serratus anterior muscles for PECS II block, and between the latissimus dorsi and serratus anterior muscles for serratus plane block. Although the local anesthetic is injected on serratus anterior muscles in both PECS II block and serratus plane block, the local anesthetic is injected to more dorsal side in serratus plane block than in PECS II block. Therefore, serratus plane block can anesthetize more

\footnotetext{
* Correspondence: tatsuya_9250@yahoo.co.jp

${ }^{1}$ Department of Anesthesia, Hokkaido Medical Center for Child Health and Rehabilitation, 1-240-6, Kanayama 1-jo, Teine-ku Sapporo-shi, Hokkaido 006-0041, Japan

Full list of author information is available at the end of the article
}

number of intercostal nerves. These blocks might be effective as alternative analgesia for the anterior thoracic wall without causing the potential serious complications reported after PVB.

Previously, we performed the clinical research to investigate optimal volume of the local anesthetic in serratus plane block [4]. In this study, we investigated the spread of the injectate by performing serratus plane injection with color dye in Thiel-embalmed cadavers and subsequent dissection.

\section{Materials and methods}

This observational study was approved by the institutional ethics committee of Sapporo Medical University School of Medicine (\#26-1-7).

Nine Thiel-embalmed cadavers were prepared for the study. Serratus plane injection was performed in the Thiel-embalmed cadavers with methylene blue dye. A 6to $15-\mathrm{MHz}$ linear array ultrasound transducer (S-Nerve, FUJIFILM-SonoSite, Tokyo, Japan) was placed on the mid-axillary line at the level of the fourth intercostal 
space. The interfascial plane between the serratus anterior and the pectoralis major muscles (the serratus plane) was identified. A 22-gauge block needle (Stimuplex Ultra, B-Braun Medical Inc., Melsungen, Germany) was inserted at the cephalad edge of the probe and advanced in-plane until the serratus plane. Once the needle tip was confirmed to be in the correct position, 20 or $40 \mathrm{ml}$ of methylene blue dye was injected into the plane. Twenty milliliters of dye was injected to the left side and $40 \mathrm{ml}$, to the other.

Dissection of the thoracic walls was performed $20 \mathrm{~min}$ after the injection. The intercostal nerves, medial and lateral pectoral nerves, the long thoracic nerve, and the thoracodorsal nerve were identified. The spread of the injectate was assessed by color-stained nerves.

\section{Results}

Nine thiel-embalmed cadavers were prepared; all were over 80 years old, four females and five males. The detail profiles including height and weight were not measured.

One hemithoracic wall in the 40-ml group was excluded because of the surgical scar. The serratus planes, fourth ribs, needles, and spread of the dye were clearly visualized in all the other 17 specimens (Fig. 1). After the anterolateral chest walls had been dissected, the spread of the dye was confirmed at the surface of the serratus anterior muscle in all cases (Fig. 2). The intramuscular spread of the dye was not observed in dissection. All T2-T5 intercostal nerves in the 40-ml group and all T3-T4 nerves in the 20-ml group were stained by the dye (Table 1). The number of stained intercostal nerves was larger in the $40-\mathrm{ml}$ group than that in the
20 -ml group ( $5 \pm 0.7$ vs. $4 \pm 1.1$ nerves, $p=0.02$ ). The degrees of spread to the medial and lateral pectoral, long thoracic, and thoracodorsal nerves were comparable between the groups.

\section{Discussion}

This comparative study showed that $40 \mathrm{ml}$ of the color dye spread to wider longitudinal directions after ultrasound-guided serratus plane injection in adult Thiel-embalmed cadavers than did $20 \mathrm{ml}$. Serratus plane block was reported to be effective in pain relief of the anterior chest wall [5, 6]. Besides, serratus plane block combined with PECS block was also reported to be effective [7-9]. T1 intercostal nerve and medial/lateral pectoral nerves were not frequently stained even when $40 \mathrm{ml}$ of dye was used in our study. Long thoracic and thoracodorsal nerves were not frequently stained.

Blanco reported that sensory loss was obtained from T2 to T9 after injection of $0.4 \mathrm{ml} / \mathrm{kg}$ of $0.125 \%$ levobupivacaine for serratus anterior block [3]. The volume of injection in the present study, $40 \mathrm{ml}$, was larger than that in their study. Nevertheless, the range of spread of color dye in our study was narrower compared to the observed effects of sensory block in Blanco's study. The difference could be because of the gap between clinical effect and anatomical evaluation of spread. Kikuchi et al. reported that the spread of the dye after PECS I and II blocks in a Thiel-embalmed cadaver extended to the mid-axillary line and to the surface of the serratus anterior muscle, respectively [10]. The result concerning serratus plane injection was similar to our study in that the dye spread over the anterolateral chest wall and in that the dye never spread into the axillary region. As
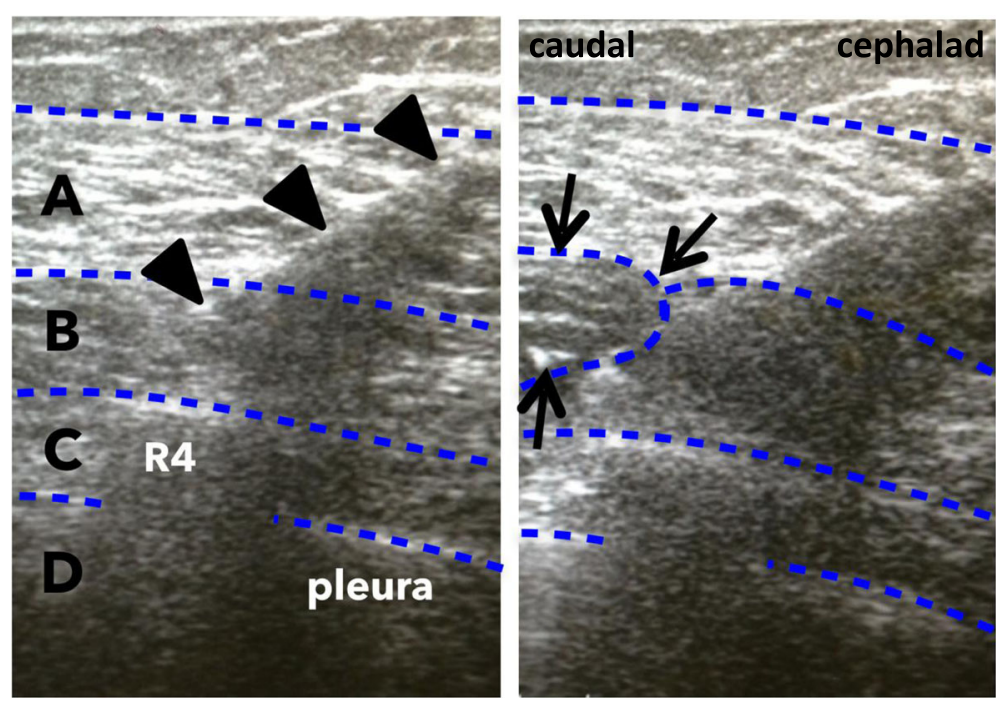

Fig. 1 Ultrasound-guided serratus plane block 

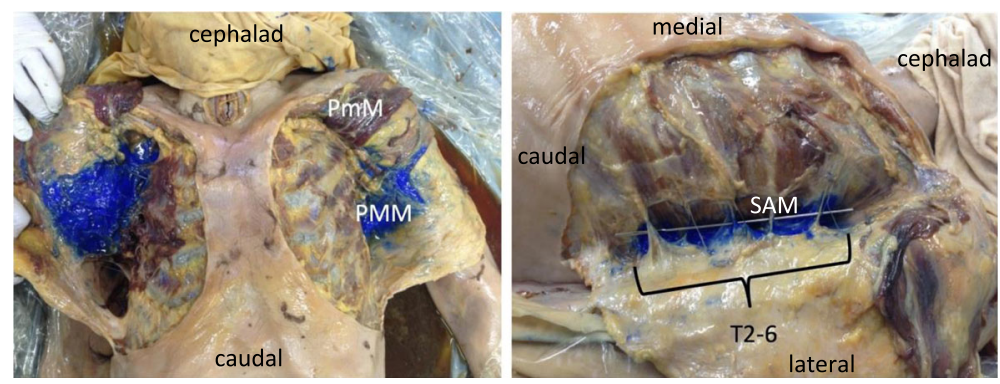

Fig. 2 Dissections after color dye injections

mentioned above, T1 intercostal nerve, medial/lateral pectoral nerves, long thoracic nerve, and thoracodorsal nerve were seldom stained. The lateral pectoral nerve originates from $\mathrm{C} 5-\mathrm{C} 7$ and runs between the pectoral major and minor muscles. The medial pectoral nerve originates from $\mathrm{C} 8-\mathrm{T} 1$ and runs under the pectoral minor muscle. The long thoracic nerve originates from $\mathrm{C} 5-\mathrm{C} 7$ and runs on the serratus anterior muscle. The thoracodorsal nerve originates from $\mathrm{C} 6-\mathrm{C} 8$ and innervates the latissimus dorsi muscle, through the posterior part of the axilla. Our study showed that the dye injected into the serratus plane never reached the axilla or dorsal aspect and spread only over the anterolateral thoracic wall. PECS I block may be more suitable for blocking pectoral nerves [10].

Our study has several limitations. First, no anatomist verified the dissections. Second, the height and weight of cadavers were not measured. Finally, it is difficult to predict the clinical effect of a block in the cadaver research.

The dye spread was confirmed after serratus plane injection in Thiel-embalmed cadavers and subsequent dissections. The range of dye spread in the longitudinal direction was greater after injection of $40 \mathrm{ml}$

Table 1 The number of nerves stained by dye

\begin{tabular}{lll}
\hline Affected nerve & $20 \mathrm{ml}(n=9)$ & $40 \mathrm{ml}(n=8)$ \\
\hline T1 & 1 & 1 \\
T2 & 3 & 8 \\
T3 & 9 & 8 \\
T4 & 9 & 8 \\
T5 & 8 & 8 \\
T6 & 3 & 5 \\
T7 & 0 & 0 \\
Lateral pectoral & 2 & 3 \\
Medial pectoral & 1 & 3 \\
Long thoracic & 3 & 4 \\
Thoracodorsal & 2 & 4
\end{tabular}

Data are expressed as absolute numbers

$\mathrm{T} 1-\mathrm{T} 7$, corresponding intercostal nerves than after $20 \mathrm{ml}$. However, a clinical study has shown that 20 and $40 \mathrm{ml}$ were not significantly different for the analgesia after radical mastectomy [4]. The clinical analgesic effect cannot necessarily be reflected by the cadaveric result of the direct staining of individual nerve branches.

\section{Funding}

There was no funding obtained for this study.

\section{Authors' contributions}

TK contributed to the study design, study conduct, data analysis, and manuscript preparation. TM contributed to the study design, data interpretation, study conduct, and manuscript preparation. SY and MY contributed to the data interpretation and manuscript review. All authors read and approved the final manuscript.

\section{Ethics approval and consent to participate}

This study protocols were approved by the institutional ethics committee of Sapporo Medical University School of Medicine.

\section{Competing interests}

The authors declare that they have no competing interests.

\section{Publisher's Note}

Springer Nature remains neutral with regard to jurisdictional claims in published maps and institutional affiliations.

\section{Author details}

${ }^{1}$ Department of Anesthesia, Hokkaido Medical Center for Child Health and Rehabilitation, 1-240-6, Kanayama 1-jo, Teine-ku Sapporo-shi, Hokkaido 006-0041, Japan. ${ }^{2}$ Department of Anesthesia, Kitami Red Cross Hospital, Kitami, Japan. ${ }^{3}$ Department of Anesthesia, Hokkaido P.W.F.A.C. Obihiro Kosei General Hospital, Obihiro, Japan. ${ }^{4}$ Department of Anesthesiology, Sapporo Medical University School of Medicine, Sapporo, Japan.

Received: 30 November 2017 Accepted: 10 January 2018

Published online: 22 January 2018

\section{References}

1. Blanco R. The 'pecs block': a novel technique for providing analgesia after breast surgery. Anaesthesia. 2011;66:847-8.

2. Blanco R, Fajardo M, Parras MT. Ultrasound description of pecs II (modified pecs I): a novel approach to breast surgery. Rev Esp Anestesiol Reanim. 2012:59:470-5.

3. Blanco R, Parras T, McDonnell JG, Prats-Galino A. Serratus plane block: a novel ultrasound-guided thoracic wall nerve block. Anaesthesia. 2013;68 1107-13.

4. Kunigo T, Murouchi T, Yamamoto S, Yamakage M. Injection volume and anesthetic effect in serratus plane block. Reg Anesth Pain Med. 2017:42:737-40

5. Madabushi R, Tewari S, Gautam SK, Agarwal A, Agarwal A. Serratus anterior plane block: a new analgesic technique for post-thoracotomy pain. Pain Physician. 2015;18:E421-4. 
6. Kunhabdulla NP, Agarwal A, Gaur A, Gautam SK, Gupta R, Agarwal A. Serratus anterior plane block for multiple rib fractures. Pain Physician. 2014; 17:E553-5.

7. Bouzinac A, Brenier G, Dao M, Delebos A. Bilateral association of pecs I block and serratus plane block for postoperative analgesia after double modified radical mastectomy. Minerva Anestesiol. 2015;81:589-90.

8. Fusco P, Scimia P, Marinangeli F, Pozone T, Petrucci E. The association between the ultrasound guided serratus plane block and pecs I block can represent a valid alternative to conventional anesthesia in breast surgery in a seriously ill patient. Minerva Anestesiol. 2016;82:241-2.

9. Fujiwara S, Komasawa N, Minami T. Pectral nerve blocks and serratusintercostal plane block for intractable postthoracotomy syndrome. J Clin Anesth. 2015;27:275-6.

10. Kikuchi M, Tanaki S, Nomura T, Goto T. Difference in the spread of injectate between ultrasound-guided pectoral nerve blocks I and II. A cadaver study (in Japanese with English abstract). Masui (Jpn J Anesthesiol). 2016;65:314-7.

\section{Submit your manuscript to a SpringerOpen ${ }^{\bullet}$ journal and benefit from:}

- Convenient online submission

- Rigorous peer review

- Open access: articles freely available online

- High visibility within the field

- Retaining the copyright to your article

Submit your next manuscript at $\gg$ springeropen.com 